Pacific Journal of Mathematic 


\section{THE HIGHER ORDER DIFFERENTIABILITY OF SOLUTIONS OF ABSTRACT EVOLUTION EQUATIONS}

\section{P. Suryanarayana}

In this paper, the regularity of the solution of the initial value problem for the abstract evolution equation

$$
\frac{d u}{d t}+A(t) u=f(t), \quad u(0) \in X, \quad 0 \leqq t \leqq T
$$

and the associated homogeneous equation

$$
\frac{d u}{d t}+A(t) u=0, \quad u(0) \in X, \quad 0 \leqq t \leqq T
$$

in a Banach space $X$ is considered. Here $u=u(t)$ and $f(t)$ are functions from $[0, T]$ to $X$ and $A(t)$ is a function on $[0, T]$ to the set of (in general) unbounded linear operators acting in $X$.

Definition. $u(t)$ is called a strict solution of $(0.1)$ or $(0.2)$ in $(s, T]$ if

(i) $u(t)$ is strongly continuous in the closed interval $[s, T]$ and is strongly continuously differentiable in the semiclosed interval $(s, T]$,

(ii) $u(t) \in D(A(t))$, the domain of $A(t)$, for each $t \in(s, T]$,

(iii) $u(t)$ satisfies $(0.1)$ resp. $(0.2)$ in $(s, T], u(s)$ coinciding with the given initial value at $t=s$.

It is assumed that $A(t)$ for each $t \in[0, T]$ satisfies the following conditions.

(i) $-A(t)$ generates a semigroup $\exp (-s A(t))$ of operators analytic in the sector $|\arg s|<\theta, s \neq 0,0<\theta<\pi / 2$,

(ii) For any complex number $\lambda$ satisfying $|\arg \lambda|<\pi / 2+\theta$, $0<\theta<\pi / 2,(\partial / \partial t)(\lambda+A(t))^{-1}$ exists in the operator topology and that there exist constants $N$ and $\rho$ independent of $t$ and $\lambda$ with $N>0,0 \leqq \rho<1$ such that

$$
\left\|\frac{\partial}{\partial t}(\lambda+A(t))^{-1}\right\| \leqq N|\lambda|^{\rho-1}
$$

The main result proved in the paper can be stated as follows. If, in addition to the above assumptions, $A(t)^{-1} \in C^{n+\alpha}[0, T]$ in the uniform operator topology, $B(t)$, a bounded operator for each $t \in[0, T]$ is of class $C^{n-1+\beta}[0, T]$, and $f(t) \in C^{n-1+\gamma}[0, T]$ in the strong topology, then the unique strict solution $u(t)$ of

$$
\frac{d u}{d t}+(A(t)+B(t)) u=f(t), \quad u(0) \in X . \quad 0 \leqq t \leqq T
$$

belongs to the class $C^{n+\delta}\left[s_{0}, T\right], s_{0}>0$ arbitrary, $\delta>0$ depending on $\alpha, \beta, \gamma$ and $\rho$. In this no assumption regarding the constancy of the domain $D(A(t))$ is made. 
From the above it is clear that if further $A(t)^{-1} \subseteq C^{\infty}[0, T]$, $B(t) \in C^{\infty}[0, T]$ and $f(t) \in C^{\infty}[0, T]$, then $u(t) \in C^{\infty}(0, T]$. It is shown by an example that the solution $u(t)$ need not be real analytic even though $A(t)^{-1}$ is real analytic and satisfies all other requirements.

The existence and uniqueness of strict solutions are established under varying hypotheses in a number of papers, Kato [3, 4, 5, 7], Tanabe [10,11, 12], Kato and Tanabe [8] and Fisher [1] based on the theory of semigroups of operators. A survey of work done on the abstract evolution equation (0.1) is given in Kato [5]. Kato and Tanabe [8] established the existence and uniqueness of strict solutions without any assumptions on the constancy of the domain of the operators $A(t)$. They also proved that the solution $u(t)$ is analytic when $(-A(t))$ is a generator of an analytic semigroup for complex values of $t$ in a convex neighbourhood of $[0, T]$ provided that the inhomogeneous term $f(t)$ is also analytic. On the other hand, when $D(A(t))$ is constant, Tanabe [12] proved that the solution of $(0.2)$ is twice differentiable if $A(t) A(s)^{-1}$ is Holder continuously differentiable. P. E. Sobolevskii [9] showed that if

$$
A(t) A(s)^{-1} \in C^{n+\varepsilon}[0, T], \quad f(t) \in C^{n}[0, T],
$$

then $u(t) \in C^{n+1}[0, T]$ and that $u(t)$ is real analytic if $A(t) A(0)^{-1}$ is real analytic.

The following notations are used throught the paper. $X$ denotes a fixed Banach space. $\Sigma$ denotes the closed sector in the complex plane consisting of the complex numbers $\lambda$ satisfying

$$
|\arg \lambda| \leqq \pi / 2+\theta, \quad 0<\theta<\pi / 2 .
$$

E.1. For each $t \in[0, T], A(t)$ is a densely defined closed linear operator acting in $X$. The resolvent set $\rho(-A(t))$ of $-A(t)$ contains $\Sigma$. The resolvent of $(-A(t))$ satisfies

$$
\left\|(\lambda+A(t))^{-1}\right\| \leqq \frac{M}{|\lambda|} \quad \text { for any } \lambda \in \Sigma, t \in[0, T],
$$

$M$ being a constant independent of $t$ and $\lambda$. (This implies that for each $t,-A(t)$ generates a semigroup $\exp (-s A(t))$ analytic in the sector $|\arg s| \leqq \theta, s \neq 0$. Hille-Phillips [2], Yosida [13]).

E.2.n. $A(t)^{-1}$ as a bounded operator for each $t \in[0, T]$ belongs to the class $C^{n+\alpha}[0, T]$ in the uniform operator topology (i.e. $d^{n} A(t)^{-1} / d t^{n}$ exists in the uniform operator topology and is Hölder continuous in the same topology with a Hölder exponent $\alpha>0$ ). 
E.3. (K-T-condition) For any $\lambda \in \Sigma, t \in[0, T]$, there exist constants $N$ and $\rho$ independent of $t$ and $\lambda$ with $N>0,0 \leqq \rho<1$ such that

$$
\left\|\frac{\partial}{\partial t}(\lambda+A(t))^{-1}\right\| \leqq \frac{N}{|\lambda|^{1-\rho}} .
$$

E.4.n. The inhomogeneous term $f(t)$ is of class $C^{n-1+r}[0, T]$ in the strong topology for $X, 0<\gamma<1$.

E.5.n. $B(t)$ for each $t \in[0, T]$ is a bounded operator and belongs to the class $C^{n-1+\beta}[0, T]$ in the uniform operator topology $(0<\beta<1)$.

We first observe that if $(d / d t) A(t)^{-1}$ exists and $\lambda \in \rho(-A(t))$, then $(d / d t)(\lambda+A(t))^{-1}$ exists and

$$
\begin{aligned}
\frac{d}{d t}(\lambda & +A(t))^{-1} \\
& =\left[1-\lambda(\lambda+A(t))^{-1}\right] \frac{d}{d t} A(t)^{-1}\left[1-\lambda(\lambda+A(t))^{-1}\right] .
\end{aligned}
$$

So $K-T$ condition always makes sense if $A(t)$ satisfies at least E.2.1 and we will always be taking $K-T$ condition in conjunction with E.2.1 at least.

We are now in a position to state our main results.

THeOREM 1. Let $A(t)$ satisfy E1, E.2.1, and E3, $B(t)$ satisfy E.5.1 and $f(t)$ satisfy E.4.1. Then the unique strict solution $u(t)$ of

$$
\frac{d u}{d t}+(A(t)+B(t)) u=f(t), \quad u(0) \in X, \quad 0 \leqq t \leqq T
$$

belongs to the class $C^{1+\varepsilon}\left[s_{0}, T\right], s_{0}>0$ arbitrary, $\delta>0$ depending on $\alpha, \beta, \gamma$ and $\rho$.

THEOREM 2. Let $A(t)$ satisfy E.1, E.2.n, and E.3, $B(t)$ satisfy E.5.n and $f(t)$ satisfy E.4.n. Then the unique strict solution $u(t)$ of (1.4) is of class $C^{n+s}\left[s_{0}, T\right], s_{0}>0$ arbitrary, $\delta>0$ depending on $\alpha, \beta, \gamma$ and $\rho$.

CoROLlary 1. If $A(t)$ satisfies E.1, E.3 and further

$$
\begin{gathered}
A(t)^{-1} \in C^{\infty}[0, T], \\
B(t) \in C^{\infty}[0, T]
\end{gathered}
$$

in the uniform operator topology and 


$$
f(t) \in C^{\infty}[0, T]
$$

in the strong topology of $X$, then the unique strict solution $u(t)$ of (1.4) is of class $C^{\infty}(0, T]$ in the strong topology of $X$.

Corollary 2. Let us assume that $D(A(t))$ is independent of $t$, $A(t)$ satisfies E.1 and E.2.n and $f(t)$ satisfies E.4.n. Further let the bounded operator $A(t) A(s)^{-1}$ be once continuously differentiable in the uniform operator topology in $t \in[0, T]$ for any fixed $s \in[0, T]$. Then the unique strict solution $u(t)$ of $(0.1)$ is of class $C^{n+8}\left[s_{0}, T\right]$ in the strong topology of $X, s_{0}>0$ arbitrary.

2. Preliminaries and known results. We collect below some results from Kato and Tanabe [8] which will be used here very frequently.

Theorem A (Kato and Tanabe). Let $A(t)$ satisfy E.1, E.2.1 and E.3 and $f(t)$ satisfy E.4.1. Then the equation (0.1) has a unique strict solution $u(t)$ given by

$$
u(t)=U(t, 0) u(0)+\int_{0}^{t} U(t, \sigma) f(\sigma) d \sigma .
$$

Here $U(t, s)$ is a bounded operator and is called evolution operator, Green's operator, propogator or fundamental solution. It is constructed as

$$
\begin{aligned}
U(t, s)= & \exp (-(t-s) A(t)) \\
& +\int_{0}^{t} \exp (-(t-\tau) A(t)) R(\tau, s) d \tau,
\end{aligned}
$$

$R(t, s)$ being determined as the solution of the integral equation

$$
R(t, s)-\int_{s}^{t} R_{1}(t, \tau) R(\tau, s) d \tau=R_{1}(t, s)
$$

where

$$
R_{1}(t, s)=-\left(\frac{\partial}{\partial t}+\frac{\partial}{\partial s}\right) \exp (-(t-s) A(s))
$$

This $U(t, s)$ has the properties

(i) $U(s, s)=I$ (The identity operator) for any $s \in[0, T]$

(ii) $U(t, r) U(r, s)=U(t, s), 0 \leqq s \leqq r \leqq t \leqq T$

(iii) The range of $U(t, s)$ is contained in $D(A(t))$ and 


$$
\begin{aligned}
\frac{\partial}{\partial t} U(t, s)= & -A(t) U(t, s) \\
= & A(t) \exp (-(t-s) A(t))-R(t, s) \\
& +\int_{s}^{t} A(t) \exp (-(t-\tau) A(t))(R(\tau, s)-R(t, s)) d \tau \\
& +\exp (-(t-s) A(t)) R(t, s) .
\end{aligned}
$$

LEMMA 1. Under the same assumptions as above, the following are true.

(a) $\quad\left\|\frac{\partial}{\partial t} \exp (-(t-s) A(t))\right\| \leqq C(t-s)^{-1}$.

$$
\text { (b) } \quad\left\|\frac{\partial}{\partial s} \exp (-(t-s) A(t))\right\| \leqq C(t-s)^{-1} \text {. }
$$

$$
\left\|R_{1}(t, s)\right\| \leqq C(t-s)^{-\rho} \text {. }
$$

$$
\|R(t, s)\| \leqq C(t-s)^{-\rho} \text {. }
$$

(e) For $0 \leqq s<\tau<t \leqq T$,

$$
\begin{aligned}
& \|R(t, s)-R(\tau, s)\| \\
& \leqq C\left\{\frac{t-\tau}{(t-s)(\tau-s)^{\rho}}+\frac{(t-\tau)^{\alpha}}{t-s}\right. \\
& \left.\quad+\frac{(t-\tau)^{1-\rho}}{(t-s)^{\rho}}+\frac{(t-\tau)^{\alpha}}{(t-s)^{\rho}} \log \frac{t-s}{t-\tau}\right\} .
\end{aligned}
$$

(f) For $0 \leqq s<\tau<t \leqq T$,

$$
\left\|R_{1}(t, s)-R_{1}(\tau, s)\right\| \leqq C\left\{\frac{t-\tau}{(t-s)(\tau-s)^{\rho}}+\frac{(t-\tau)^{\alpha}}{t-s}\right\} .
$$

(g) Let

$$
W(t, s)=\int_{s}^{t} \exp (-(t-\tau) A(t)) R(\tau, s) d \tau .
$$

Then we have

$$
\begin{aligned}
\frac{\partial}{\partial t} W(t, s)= & \int_{s}^{t} \frac{\partial}{\partial t} \exp (-(t-\tau) A(t))(R(\tau, s)-R(t, s)) d \tau \\
& -\int_{s}^{t} R_{1}(t, \tau) d \tau R(t, s) \\
& \quad+\exp (-(t-s) A(t)) R(t, s) .
\end{aligned}
$$


( h )

$$
\left\|\frac{\partial}{\partial t} W(t, s)\right\| \leqq C\left\{(t-s)^{-\rho}+(t-s)^{\alpha-1}\right\} .
$$$$
\frac{\partial}{\partial t} \int_{s}^{t} \exp (-(t-\sigma) A(t)) f(\sigma) d \sigma
$$

$$
\begin{aligned}
=\int_{s}^{t} \frac{\partial}{\partial t} \exp (-(t-\sigma) A(t))(f(\sigma)-f(t)) d \sigma \\
\quad-\int_{s}^{t} R_{1}(t, \sigma) f(t) d \sigma+\exp (-(t-s) A(t)) f(t) .
\end{aligned}
$$

Note. Throughout this section and the following, $C$ denotes a positive constant depending only on the fundamental constants $M, N$, $\theta, \rho, \alpha$ and those which appear in the assumptions of Theorem 1 . The constant $C$ is not necessarily the same at every occurence. We use $C_{\varepsilon}$ to denote a constant depending on $\varepsilon>0$ in addition to the constants mentioned above.

We also require a slightly weaker form of Theorem 6.1 [Kato and Tanabe [8]. We present it as

Theorem B (Kato and Tanabe). Let A(t) satisfy E.1, E.2.1, and E.3, $B(t)$ satisfy E.5.1 and $f(t)$ satisfy E.4.1. Then the equation (1.4) has a unique strict solution given by

$$
u(t)=U_{1}(t, 0) u(0)+\int_{0}^{t} U_{1}(t, \sigma) f(\sigma) d \sigma
$$

where

$$
U_{1}(t, s)=U(t, s)-\int_{s}^{t} U(t, \sigma) B(\sigma) U_{1}(\sigma, s) d \sigma
$$

$U(t, s)$ being the evolution operator corresponding to $(0.1)$.

We now proceed to give the proofs of theorems stated in $\S 1$. Section 3 will be devoted for the proof of Theorem 1 and $\S 4$ for Theorem 2.

For the proof of Theorem 2, we need the following Theorem $\mathrm{C}$ from Kato [6], which is the same as Lemma 13.7.1 in HillePhillips [2].

Definition 1. $H(\omega, 0)$ is the set of all densely defined closed linear operators $T$ in a Banach space $X$ satisfying

(i) the resolvent set $\rho(-T)$ contains a sector

$$
|\arg \xi| \leqq \frac{\pi}{2}+\omega, \quad 0<\omega<\frac{\pi}{2}
$$


(ii) for any $\varepsilon>0$,

$$
\left\|(T+\xi)^{-1}\right\| \leqq \frac{M_{\varepsilon}}{|\xi|} \quad \text { for }|\arg \xi| \leqq \frac{\pi}{2}+\omega-\varepsilon
$$

with $M_{\varepsilon}$ independent of $\xi$.

Definition 2. $H(\omega, \beta), \beta$ real, is the set of operators $T$ of the form $T=T_{0}-\beta$ with $T_{0} \in H(\omega, 0)$.

Theorem C. Let $T \in H(\omega, \beta)$ and let $A$ be relatively bounded with respect to $T$ so that

$$
\|A u\| \leqq a\|u\|+b\|T u\|, \quad u \in D(T) \subset D(A) .
$$

For any $\varepsilon>0$, there exists $a \beta^{\prime}>0, \delta>0$ depending on $T, \omega$ and $\varepsilon$ only, such that $T+A \in H\left(\omega-\varepsilon, \beta^{\prime}\right)$ whenever $a<\delta, b<\delta$. If in particular $\beta=0$ and $a=0$, then $T+A \in H(\omega-\varepsilon, 0)$.

3. Proof of Theorem 1. In view of Theorem B, we have only to prove the Hölder continuity of $d u / d t$. We do this in several steps.

Step $I$. We consider the solution $u(t)$ of the homogeneous equation (0.2) with the same assumptions on $A(t)$ as in Theorem 1.

Let $0 \leqq s<r<t \leqq T$.

As $(\partial / \partial t) U(t, s)=-A(t) U(t, s),(t>s)$ it is enough to estimate

$$
\|A(t) U(t, s)-A(r) U(r, s)\| \text {. }
$$

From (2.3) we have

$$
\begin{aligned}
& A(t) U(t, s)-A(r) U(r, s) \\
& =[-R(t, s)+R(r, s)] \\
& \quad+[A(t) \exp (-(t-s) A(t))-A(r) \exp (-(r-s) A(r))] \\
& \quad+[\exp (-(t-s) A(t)) R(t, s)-\exp (-(r-s) A(r)) R(r, s)] \\
& +\left[\int_{s}^{t} A(t) \exp (-(t-\tau) A(t))(R(\tau, s)-R(t, s)) d \tau\right. \\
& \left.\quad-\int_{s}^{r} A(r) \exp (-(r-\tau) A(r))(R(\tau, s)-R(r, s)) d \tau\right] \\
& =(\mathrm{i})+(\mathrm{ii})+(\mathrm{iii})+(\mathrm{iv}) \quad \text { (say) }
\end{aligned}
$$

$\|$ (i) $\|$ is estimated by (2.8). 


$$
\text { (ii) } \begin{aligned}
& =\frac{\partial}{\partial s}\{\exp (-(t-s) A(t))-\exp (-(r-s) A(r))\} \\
& =\frac{1}{2 \pi i} \int_{\Gamma} \lambda\left\{e^{(t-s) \lambda}(\lambda+A(t))^{-1}-e^{(r-s) \lambda}(\lambda+A(r))^{-1}\right\} d \lambda
\end{aligned}
$$

where $\Gamma$ is a smooth contour running in $\Sigma$ from $\infty e^{-i((\pi / 2)+\theta)}$ to $\infty e^{i((\pi / 2)+\theta)}$.

$$
\begin{aligned}
= & \frac{1}{2 \pi i} \int_{\Gamma} e^{(t-s) \lambda} \lambda \int_{r}^{t} \frac{\partial}{\partial \sigma}(\lambda+A(\sigma))^{-1} d \sigma d \lambda \\
& +\frac{1}{2 \pi i} \int_{\Gamma} \int_{r-s}^{t-s} \frac{\partial}{\partial \sigma}\left(e^{\sigma \lambda}\right) d \sigma \lambda(\lambda+A(r))^{-1} d \lambda .
\end{aligned}
$$

Using (1.1) and (1.2) we have

$$
\begin{aligned}
& \| \text { (ii) } \| \leqq C\left\{\frac{t-r}{(t-s)^{1+\rho}}+\frac{t-r}{(t-s)(r-s)}\right\} . \\
\text { (iii) }= & \{\exp (-(t-s) A(t))-\exp (-(r-s) A(r))\} R(t, s) \\
& +\exp (-(r-s) A(r))(R(t, s)-R(r, s)) \\
= & (\text { iii a) }+(\text { iii b) } \quad \text { (say). }
\end{aligned}
$$

$\|$ (iii) $a \| \leqq C(t-r) /(t-s)^{\rho}(r-s)$ using (2.7) and (2.4).

$\|$ (iii b) $\|$ is estimated by (2.8) since $\|\exp (-(r-s) A(r))\| \leqq M$.

$$
\begin{aligned}
\text { (iv) }= & \int_{s}^{r}\{A(t) \exp (-(t-\tau) A(t))-A(r) \exp (-(r-\tau) A(r))\} \\
& \quad \times\{R(\tau, s)-R(r, s)\} d \tau \\
& +\int_{s}^{r} A(t) \exp (-(t-\tau) A(t))(R(r, s)-R(t, s)) d \tau \\
& +\int_{r}^{t} A(t) \exp (-(t-\tau) A(t))(R(\tau, s)-R(t, s)) d \tau \\
= & (\text { iv a })+(\text { iv b })+(\text { iv c }) .
\end{aligned}
$$

Using (3.1) and (2.8) we have

$$
\begin{aligned}
& \|\operatorname{iv}(\mathrm{a})\|<C \int_{s}^{r}(t-r)\left\{\frac{1}{(t-\tau)^{\rho+1}}+\frac{1}{(r-\tau)(t-\tau)}\right\} \\
& \quad \times\left\{\frac{r-\tau}{(r-s)(\tau-s)^{\rho}}+\frac{(r-\tau)^{\alpha}}{r-s}+\frac{(r-\tau)^{1-\rho}}{r-s}+\frac{(r-\tau)^{\alpha}}{(r-s)^{\rho}} \log \frac{r-s}{r-\tau}\right\} d \tau .
\end{aligned}
$$

Estimating the various integrals on the right, with $\varepsilon>0$ arbitrarily chosen, we can prove

$$
\| \text { (iv a) } \|<C_{\varepsilon}\left\{\frac{(t-r)^{1-\rho}}{(r-s)^{\rho-\alpha}}+\frac{(t-r)^{1-\varepsilon}}{(r-s)^{1+\rho-\varepsilon}}+\frac{(t-r)^{\alpha-\varepsilon}}{(r-s)^{\rho-\varepsilon}}+\frac{(t-r)^{1-\rho-\varepsilon}}{(r-s)^{\rho-\varepsilon}}\right\} .
$$




$$
\begin{aligned}
\|(\mathrm{iv} \mathrm{b})\| & \leqq\|R(t, s)-R(r, s)\| \int_{s}^{r} \frac{d \tau}{t-\tau} \\
& =\|R(t, s)-R(r, s)\| \log \frac{t-s}{t-r}
\end{aligned}
$$

and this can be estimated by (2.8).

$$
\begin{aligned}
\|\left(\text { iv c) }\left\|\leqq C \int_{r}^{t} \frac{d \tau}{t-\tau}\right\| R(\tau, s)-R(t, s) \| d \tau\right. & \text { using } \\
\leqq & \left\{\frac{t-r}{(t-s)(r-s)^{\rho}}+\frac{(t-r)^{\alpha}}{t-s}\right. \\
& \left.\quad+\frac{(t-r)^{1-\rho}}{(t-s)^{\rho}}+\frac{(t-r)^{\alpha}}{(t-s)^{\rho}}\left(\frac{1}{\alpha^{2}}+\frac{1}{\alpha} \log \frac{t-s}{t-r}\right)\right\}
\end{aligned}
$$

using (2.8) and estimating the respective integrals. Combining all these estimates, we note for $0 \leqq s<s_{0} \leqq r<t \leqq T$,

$$
\|A(t) U(t, s)-A(r) U(r, s)\| \leqq C(t-r)^{\eta},
$$

$\eta=\min (1-\rho-\varepsilon, \alpha-\varepsilon)$ and $C$ depends on $s_{0}>s, s, \varepsilon$ and $T$. $\varepsilon$ can be chosen to make $\eta>0$.

This establishes the Hölder continuity of the derivative of the solution of the equation (0.2) in every interval of the form $\left[s_{0}, T\right]$, $0<s_{0}<T$.

Step II. We now consider the solution $u(t)$ of the equation (0.1) with the same assumptions on $A(t)$ and $f(t)$ as in Theorem 1.

The solution of $(0.1)$ is given by:

$$
U(t)=U(t, s) u(s)+\int_{s}^{t} U(t, \sigma) f(\sigma) d \sigma, \quad 0 \leqq s<t \leqq T,
$$

$u(s)$ being the initial value at $t=s$ and $U(t, s)$ the corresponding evolution operator.

In view of the result proved in Step I, it is enough to consider the case $u(s)=0$.

Let $0 \leqq s<r<t \leqq T$.

From the defining equations of $U(t, s)$ and $W(t, s)$ on using (2.11), (2.12) and (2.13) we obtain

$$
\begin{aligned}
\frac{d u}{d t}= & \int_{s}^{t} \frac{\partial}{\partial t} \exp \{-(t-\sigma) A(t)\}(f(\sigma)-f(t)) d \sigma \\
& -\int_{s}^{t} R_{1}(t, \sigma) f(t) d \sigma+\exp (-(t-s) A(t)) f(t) \\
& +\int_{s}^{t} \frac{\partial}{\partial t} W(t, \sigma) f(\sigma) d \sigma
\end{aligned}
$$


Using the Hölder continuity of $f(t)$, the estimates (2.4) through (2.13) and the estimates obtained in Step I, we can prove after some tedious computations that for $0 \leqq s<s_{0} \leqq r<t \leqq T$,

$$
\left\|\frac{d u(t)}{d t}-\frac{d u(r)}{d r}\right\|<K(t-r)^{\eta}
$$

where $\eta=\min \{1-\rho-\varepsilon, \alpha-\varepsilon, \gamma-\varepsilon\}, \varepsilon>0$ being arbitrarily chosen to make $\eta>0 . K$ is a constant depending on $s_{0}, s, \varepsilon$ and $T$ but not on $t$ and $r$. This establishes the Hölder continuity of the derivative of the solution of $(0.1)$ in every interval of the form $\left[s_{0}, T\right]$, $0<s_{0}<T$.

Step III. The existence and uniqueness of the solution of the equation (1.4) is established in Theorem B. We have only to establish the Hölder continuity of the derivative $d u / d t$ of this solution. Because $u(t) \in C^{1}(0, T]$ and $B(t)$ is Hölder continuous, we have that $B(t) u(t) \in C^{\beta}(0, T]$. We can treat $u(t)$ as the solution of the equation

$$
\frac{d u}{d t}+A(t) u(t)=f(t)-B(t) u(t), \quad u_{0} \in X \text { given } .
$$

As $A(t)$ satisfies the conditions used in Step II and $f(t)-B(t) u(t)$ is strongly Hölder continuous with Hölder exponent $\min (\gamma, \beta)$, by appealing to the result in Step II, we have $u(t) \in C^{1+\eta}\left[s_{0}, T\right]$,

$$
\eta=\min (1-\rho-\varepsilon, \alpha-\varepsilon, \gamma-\varepsilon, \beta-\varepsilon), \quad s_{0}>0,
$$

$\varepsilon>0$ chosen arbitrarily to make $\eta>0$.

This completes the proof of Theorem 1.

4. Proof of Theorem 2. The proof of Theorem 2 will be given after a few preparatory lemmas.

Let us first remark that if $u(t)$ is a strict solution of

$$
\frac{d u}{d t}+(A(t)+B(t)) u=f(t), \quad u(0) \in X, \quad 0 \leqq t \leqq T,
$$

then $e^{-\boldsymbol{K} t} u(t)$ is the strict solution of

$$
\frac{d u}{d t}+(A(t)+B(t)+K) u=e^{-\boldsymbol{K} t} f(t), \quad u(0) \in X, \quad 0 \leqq t \leqq T
$$

and conversely. So we may, if necessary, consider the equation (4.1) instead of (1.4) with a suitable choice of $K$. 
operator and E.3 holds, then for a suitable $K>0$,

$$
A(t)+K+\left\{\frac{d}{d t}(A(t)+K)^{-1}\right\}(A(t)+K) \quad\{=A(t ; K) \text { for short }\}
$$

satisfies E.1 with a possibly different constant $M$.

Proof. We can regard $-A(t ; K)$ as a perturbation of the analytic semi-group generator $-(A(t)+K)$. If $u \in D(A(t))$, we have

$$
\left\|\frac{d}{d t}(A(t)+K)^{-1}(A(t)+K) u\right\| \leqq\left\|\frac{d}{d t}(A(t)+K)^{-1}\right\|\|(A(t)+K) u\| .
$$

So $(d / d t)(A(t)+K)^{-1}(A(t)+K)$ is relatively bounded with respect to $(A(t)+K)$ with a relative bound

$$
\leqq\left\|\frac{d}{d t}(A(t)+K)^{-1}\right\|
$$

According to Theorem $\mathrm{C}$ of $\S 2,-A(t ; K)$ generates an analytic semigroup if we can make

$$
\left\|\frac{d}{d t}(A(t)+K)^{-1}\right\|<\frac{1}{1+M}
$$

$M$ being the constant appearing in E.1. In view of $K-T$ condition we have

$$
\left\|\frac{d}{d t}(A(t)+K)^{-1}\right\| \leqq \frac{N}{|K|^{1-\rho}}
$$

uniformly for all $t \in[0, T]$. So if we choose $K>0$ such that

$$
N K^{\rho-1}<(1+M \operatorname{Sec} \theta)^{-1}
$$

(the term $\operatorname{Sec} \theta$ is introduced for convenience in work later on), we have for each $t \in[0, T],-A(t ; K)$ generates an analytic semigroup. Further, the resolvent set of each of these operators contains the sector $\Sigma$ and

$$
\left\|(\lambda+A(t ; K))^{-1}\right\| \leqq \frac{M^{1}}{|\lambda|}
$$

for $\lambda \in \Sigma$,

$M^{1}$ being a constant independent of $t$ and $\lambda$. This completes the proof of Lemma 2.

Lemma 3. If $A(t)$ satisfies E.3 and E.2.n, then for a suitable $K>0, A(t ; K)$ satisfies E.2.n-1. 
Proof. $A(t ; K)=\left\{1+(d / d t)(A(t)+K)^{-1}\right\}(A(t)+K)$. In view of E.3, we can choose $K>0$ such that

$$
\left\|\frac{d}{d t}(A(t)+K)^{-1}\right\|<1
$$

so that $\left\{1+(d / d t)(A(t)+K)^{-1}\right\}^{-1}$ exists as a bounded operator. Then for such a choice of $K$,

$$
A(t ; K)^{-1}=(A(t)+K)^{-1}\left\{1+\frac{d}{d t}(A(t)+K)^{-1}\right\}^{-1}
$$

Also in view of (1.3) and $A(t)^{-1} \in C^{n+\alpha}[0, T]$, it follows that

$$
A(t ; K)^{-1} \in C^{n-1+\alpha}[0, T] \text {. }
$$

Hence the Lemma is proved.

Lemma 4. If $A(t)$ satisfies E.1, E.2.2 and E.3, then for $a$ suitable $K>0, A(t ; K)$ satisfies E.3 with a possibly different $N$ but with the same $\rho(0 \leqq \rho<1)$.

Proof. By Lemma $1, A(t ; K)$ satisfies E.1 if $K>0$ is chosen according to (4.2). Let $\lambda \in \Sigma$. From the second resolvent equation, we have

$$
\begin{aligned}
(\lambda+ & A(t ; K))^{-1}-(\lambda+A(t)+K)^{-1} \\
& =-(\lambda+A(t ; K))^{-1} \frac{d}{d t}(A(t)+K)^{-1}(A(t)+K)(\lambda+A(t)+K)^{-1} .
\end{aligned}
$$

Since $A(t)$ satisfies E.2.2, $(\partial / \partial t)(A(t ; K)+\lambda)^{-1}$ exists for $\lambda \in \Sigma$ noting (1.3). From (4.4) we have

$$
\begin{aligned}
\frac{\partial}{\partial t}(A(t ; K)+\lambda)^{-1} \\
=\frac{\partial}{\partial t}(\lambda+K+A(t))^{-1} \\
\quad-(\lambda+A(t ; K))^{-1} \frac{d^{2}}{d t^{2}}(A(t)+K)^{-1}(A(t)+K)(A(t)+\lambda+K)^{-1} \\
\quad-(\lambda+A(t ; K))^{-1} \frac{d}{d t}(A(t)+K)^{-1} \frac{\partial}{\partial t}\left\{(A(t)+K)(\lambda+K+A(t))^{-1}\right\} \\
\quad-\frac{\partial}{\partial t}(A(t ; K)+\lambda)^{-1} \frac{d}{d t}(A(t)+K)^{-1}(A(t)+K)(A(t)+\lambda+K)^{-1} \\
=(1)+(2)+(3)+(4) \quad \text { say . }
\end{aligned}
$$




\section{Therefore}

$$
\left\|\frac{\partial}{\partial t}(A(t ; K)+\lambda)^{-1}\right\| \leqq\|(1)\|+\|(2)\|+\|(3)\|+\|(4)\| .
$$

Now

$$
\begin{array}{rr}
\|(1)\| \leqq \frac{N}{|\lambda+K|^{1-\rho}} & \text { in view of E.3. } \\
\|(2)\| \leqq & \| A(t ; K)+\lambda)^{-1} \| \\
\times\left\|\frac{d^{2}}{d t^{2}}(A(t)+K)^{-1}\right\|\left\|(A(t)+K)(A(t)+\lambda+K)^{-1}\right\| & \text { using E.1 }
\end{array}
$$

where

$$
C_{K}=\operatorname{Sup}_{0 \leqq t \leqq T}\left\|\frac{d^{2}}{d t^{2}}(A(t)+K)^{-1}\right\|
$$

which is finite in view of E.2.2. Further from (4.4) we have

$$
\begin{aligned}
\| & (A(t ; K)+\lambda)^{-1} \| \\
\leqq & \left\|(A(t)+K+\lambda)^{-1}\right\| \\
& +\left\|(A(t ; K)+\lambda)^{-1}\right\|\left\|\frac{d}{d t}(A(t)+K)^{-1}\right\|\left\|1-\lambda(A(t)+\lambda+K)^{-1}\right\| \\
\leqq & \frac{M}{|\lambda+K|}+\left\|(A(t ; K)+\lambda)^{-1}\right\| \frac{N}{K^{1-\rho}}\left(1+M \frac{|\lambda|}{|\lambda+K|}\right) \\
\leqq & \frac{M}{|\lambda+K|}+\left\|(A(t ; K)+\lambda)^{-1}\right\| \frac{N}{K^{1-\rho}}(1+M \operatorname{Sec} \theta) \\
\leqq & \frac{M}{|\lambda+K|}+\left\|(A(t ; K)+\lambda)^{-1}\right\| \mu, \quad \mu<1 \quad \text { (because of (4.2)). }
\end{aligned}
$$

Therefore

$$
\left\|(A(t ; K)+\lambda)^{-1}\right\|<\frac{M}{|\lambda+K|(1-\mu)} .
$$

Hence

$$
\begin{aligned}
\|(2)\|< & \frac{M C_{K}}{|\lambda+K|} \frac{(1+M \operatorname{Sec} \theta)}{1-\mu} \\
\|(3)\| \leqq & \left\|(A(t ; K)+\lambda)^{-1}\right\| \\
& \times\left\|\frac{d}{d t}(A(t)+K)^{-1}\right\|\left\|\frac{\partial}{\partial t}\left(1-\lambda(A(t)+K+\lambda)^{-1}\right)\right\| \\
< & \frac{M_{1} N^{2} \operatorname{Sec} \theta}{K^{1-\rho}|\lambda+K|^{1-\rho}} .
\end{aligned}
$$




$$
\begin{aligned}
\|(4)\| \leqq & \left\|\frac{\partial}{\partial t}(A(t ; K)+\lambda)^{-1}\right\| \\
& \quad \times\left\|\frac{d}{d t}(A(t)+K)^{-1}\right\|\left\|1-\lambda(A(t)+K+\lambda)^{-1}\right\| \\
\leqq & \left\|\frac{\partial}{\partial t}(A(t ; K)+\lambda)^{-1}\right\| \mu, \\
\mu & =\frac{N(1+M \operatorname{Sec} \theta)}{K^{1-\rho}}<1 .
\end{aligned}
$$

Combining all these estimates we have

$$
\left\|\frac{\partial}{\partial t}(A(t ; K)+\lambda)^{-1}\right\| \leqq \frac{N^{1}}{|\lambda+K|^{1-\rho}} \leqq \frac{N_{1}}{|\lambda|^{1-\rho}}
$$

$N^{1}, N_{1}$ being constants which do not depend on $t$ or $\lambda$. Thus $A(t ; K)$ satisfies E.3 with the same $\rho$ and this completes the proof of the Lemma.

Proof of Theorem 2. We wish to prove this theorem by induction. First the case $n=1$ is Theorem B of Kato and Tanabe.

So let us now assume the theorem true for $n=m$ and make the induction hypothesis that $A(t)$ satisfy E.1, E.2. $m+1$ and E.3, $B(t)$ satisfy E.5. $m+1$ and $f(t)$ satisfy E.4. $m+1$. Let $K>0$ be so chosen to satisfy (4.2) and to allow

$$
\left\{1+\frac{d}{d t}(A(t)+K)^{-1}+B(t)(A(t)+K)^{-1}\right\}^{-1} \quad\left(=B(t ; K)^{-1}\right)
$$

exist as a bounded operator for each $t \in[0, T]$. This is possible because $\operatorname{Sup}_{0 \leqq t \leqq T}\|B(t)\|$ is finite and $A(t)$ satisfies E.1 and E.2.

As remarked earlier, we will consider the equation

$$
\begin{aligned}
\frac{d u}{d t}+(A(t)+B(t)+K) u & =e^{-K t} f(t), \\
u(0) & =U_{0} \in X,
\end{aligned}
$$

with $K$ chosen above.

In view of Theorem $\mathrm{B}$, equation (4.1) has a unique strict solution under our present hypotheses on $A(t), B(t)$ and $f(t)$.

Let $F(t)=e^{-\boldsymbol{K} t} f(t)$,

$$
g(t)=\left(B(t ; K) \frac{d}{d t} B(t ; K)^{-1}\right) F(t) .
$$

In view of Lemmas 2,3 , and 4 and our induction hypothesis, we note that $A(t ; K)$ satisfies E.1, E.2.m and E.3. Also because $A(t)^{-1} \in C^{m+1+\alpha}[0, T], B(t) \in C^{m+\beta}[0, T], f(t) \in C^{m+r}[0, T]$, if follows that 
(i) $B(t)+B(t ; K)(d / d t) B(t ; K)^{-1}$ is a bounded operator for each $t \in[0, T]$,

(ii) $B(t ; K) \in C^{m+\nu}[0, T], \nu=\min (\alpha, \beta)$

(iii) $B(t)+B(t ; K)(d / d t) B(t ; K)^{-1} \in C^{m-1+2}[0, T]$,

(iv) $(d F / d t)+g(t) \in C^{m-1+\eta}[0, T], \eta=\min (\nu, \gamma)$.

So $A(t ; K), B(t)+B(t ; K)(d / d t) B(t ; K)^{-1}, g(t)$ satisfy respectively the conditions for $A(t), B(t), f(t)$ of the theorem with $n=m$.

Let $t_{0} \in(0, T)$ be arbitrarily chosen. Then consider the equation

(4.6) $\frac{d v}{d t}+\left(A(t ; K)+B(t)+B(t ; K) \frac{d}{d t} B(t ; K)^{-1}\right) v=-\left(\frac{d F}{d t}+g(t)\right)$

$0<t_{0} \leqq t \leqq T$ with initial value at $t_{0}$,

$$
v\left(t_{0}\right)=-F\left(t_{0}\right)+B\left(t_{0} ; K\right)\left(A\left(t_{0}\right)+K\right) u\left(t_{0}\right)
$$

where $u\left(t_{0}\right)$ is the value of the strict solution of (4.1) at $t=t_{0}$.

Because the equation (4.6) satisfies the conditions of theorem with $n=m$, we have that the unique strict solution $v(t)$ of $(4.6)$ is of class $C^{m+\delta}\left[t_{1}, T\right], t_{1}>t_{0}$ arbitrary.

Let $w(t)=F(t)+v(t)$.

Clearly $w(t) \in C^{m+\delta}\left[t_{1}, T\right]$. Then

$$
\begin{aligned}
(A(t)+ & K)^{-1} B(t ; K)^{-1} \frac{d w}{d t}+\left\{1+(A(t)+K)^{-1} \frac{d}{d t} B(t ; K)^{-1}\right\} w \\
= & (A(t)+K)^{-1} B(t ; K)^{-1} \frac{d F}{d t}+\left\{1+(A(t)+K)^{-1} \frac{d}{d t} B(t ; K)^{-1}\right\} F \\
& +(A(t)+K)^{-1} B(t ; K)^{-1} \\
& \times\left[\frac{d v}{d t}+B(t ; K)(A(t)+K)\left(1+(A(t)+K)^{-1} \frac{d}{d t} B(t ; K)^{-1}\right) v\right] \\
= & (A(t)+K)^{-1} B(t ; K)^{-1} \frac{d F}{d t}+\left\{1+(A(t)+K)^{-1} \frac{d}{d t} B(t ; K)^{-1}\right\} F \\
& +(A(t)+K)^{-1} B(t ; K)^{-1}\left(-\frac{d F}{d t}-g(t)\right)
\end{aligned}
$$

in view of (4.6) and noting that, $B(t ; K)(A(t)+K)=A(t ; K)+B(t)=F(t)$ by our choice of $g(t)$ (see 4.5).

Thus $w(t)$ satisfies

$$
\begin{gathered}
(A(t)+K)^{-1} B(t ; K)^{-1} \frac{d w}{d t}+\left(1+(A(t)+K)^{-1} \frac{d}{d t} B(t ; K)^{-1}\right) w=F(t), \\
t_{0} \leqq t \leqq T, \\
w\left(t_{0}\right)=F\left(t_{0}\right)+v\left(t_{0}\right)=B\left(t_{0} ; K\right)\left(A\left(t_{0}\right)+K\right) u\left(t_{0}\right) .
\end{gathered}
$$


Therefore

$$
(A(t)+K)^{-1} \frac{d}{d t}\left(B(t ; K)^{-1} w\right)+w=F(t) .
$$

Writing $\xi(t)=B(t ; K)^{-1} w$, we have

$$
\begin{array}{ll}
(A(t)+K)^{-1} \frac{d \xi}{d t}+\left\{1+\frac{d}{d t}(A(t)+K)^{-1}+B(t)(A(t)+K)^{-1}\right\} \xi=F(t), \\
\xi\left(t_{0}\right)=B\left(t_{0} ; K\right)^{-1} w\left(t_{0}\right)=\left(A\left(t_{0}\right)+K\right) u\left(t_{0}\right), & t_{0} \leqq t \leqq T .
\end{array}
$$

So

$$
\frac{d}{d t}\left((A(t)+K)^{-1} \xi\right)+(A(t)+K+B(t))(A(t)+K)^{-1} \xi=F(t) .
$$

Writing $(A(t)+K)^{-1} \xi=\zeta$, we have

$$
\begin{array}{ll}
\frac{d \zeta}{d t}+(A(t)+B(t)+K) \zeta=F(t), & \\
\zeta\left(t_{0}\right)=u\left(t_{0}\right), & t_{0} \leqq t \leqq T .
\end{array}
$$

Since (4.1) has a unique strict solution $u(t), 0<t \leqq T$, and since the unique strict solution of (4.7) coincides with that of (4.7) at $t=t_{0}$, we conclude that

$$
\zeta(t)=u(t), \quad \text { for } t \in\left[t_{0}, T\right] .
$$

Now

$$
u(t)=(A(t)+K)^{-1} \xi(t)=(A(t)+K)^{-1} B(t ; K)^{-1} w(t) .
$$

Because $w(t) \in C^{m+\delta}\left[t_{1}, T\right], B(t ; K)^{-1} \in C^{m+\nu}[0, T]$, and

$$
(A(t)+K)^{-1} \in C^{m+1+\alpha}[0, T],
$$

we have $u(t) \in C^{m+\delta}\left[t_{1}, T\right]$ and

$$
(A(t)+K) u(t)=B(t ; K)^{-1} w(t) \in C^{m+\delta}\left[t_{1}, T\right] .
$$

Therefore

$$
\frac{d u}{d t}=-(A(t)+K) u(t)-B(t) u(t) \in C^{m+\delta}\left[t_{1}, T\right] .
$$

Hence $u \in C^{m+1+\delta}\left[t_{1}, T\right]$.

Because $t_{0}>0$, and $t_{1}>t_{0}$ are arbitrary, we have

$$
u(t) \in C^{m+1+\delta}\left[t_{1}, T\right], \quad t_{1}>0
$$

arbitrary. This completes the proof of Theorem 2 .

Corollary 1 follows immediately. 
Example of an operator $A(t)$ which satisfies E.1 and E.3 with $A(t)^{-1}$ real analytic the corresponding evolution operator $U(t, s)$ of which is not real analytic:

This example is the same as given in Kato [5].

Let $X=L^{2}[a, b], 0<a<b<T . \quad A(t)$ be a family of multiplication operators in $X$ defined by

$$
A(t) u(x)=(t-x)^{-2} u(x), \quad u(x) \in D(A(t)) .
$$

These are positive and self adjoint operators and so E.1 is clearly satisfied. Because $(\partial / \partial t)(A(t)+\lambda)^{-1}$ is a multiplication operator defined by

$$
\begin{aligned}
\frac{\partial}{\partial t}(A(t)+\lambda)^{-1} u(x) & =\frac{2(t-x)^{-3}}{\left(\lambda+(t-x)^{-2}\right)^{2}} u(x), \\
\left\|\frac{\partial}{\partial t}(A(t)+\lambda)^{-1}\right\| & \leqq \operatorname{Sup}_{t, x}\left|\frac{2(t-x)^{-3}}{\left(\lambda+(t-x)^{-2}\right)^{2}}\right| \\
& \leqq 2 \operatorname{Sup}_{t, x}\left|\left\{\frac{(t-x)^{-2}}{\lambda+(t-x)^{-2}}\right\}^{3 / 2} \frac{1}{\left\{\lambda+(t-x)^{-2}\right\}^{1 / 2}}\right| \\
& \leqq \frac{C}{|\lambda|^{1 / 2}} \quad \text { for } \lambda \in \Sigma .
\end{aligned}
$$

Thus $K-T$ condition is satisfied with $\rho=1 / 2$. The evolution operator to this $A(t)$ is given by

$$
\begin{aligned}
U(t, s) u(x) & =\exp \left\{(t-x)^{-1}-(s-x)^{-1}\right\} u(x) & & \text { if } x>t \text { or } x<s \\
& =0 & & \text { if } s \leqq x \leqq t .
\end{aligned}
$$

Let

$$
\begin{aligned}
\eta(x) & =e^{-(1 / x)} & & \text { if } x>0 \\
& =0 & & \text { if } x \leqq 0 \\
U(t, s) u(x) & =\eta(x-t) \eta(s-x) u(x) . & &
\end{aligned}
$$

As $|\eta(x)| \leqq 1,\|U(t, s)\| \leqq 1$.

It is also clear that $U(t, t)=I$ and

$$
U(t, r) U(r, s)=U(t, s) \quad s \leqq r \leqq t .
$$

It can easily be shown that

$$
\operatorname{Sup}_{t, x}\left|\frac{\eta(x-t-h) \eta(s-x)-\eta(x-t) \eta(s-x)}{h}-\eta^{1}(x-t) \eta(s-x)\right|<\varepsilon
$$

for sufficiently small $h$ so that 


$$
\begin{aligned}
\frac{\partial}{\partial t} U(t, s) & =-(x-t)^{-2} U(t, s) \\
& =-A(t) U(t, s) .
\end{aligned}
$$

This implies that $U(t, s)$ is the evolution operator. Now $A(t)^{-1}$, being multiplication by $(t-x)^{2}$, is analytic in $t$.

Also $U(t, s)=0$ if $s \leqq a$ and $t \geqq b$ and $U(t, s) \neq 0$ otherwise. Hence $U(t, s)$ is not analytic in $t$.

We note that this is due to the fact that even though $A(t)^{-1}$ has an analytic extension for $t$ complex, $-A(t)$ is not the generator of an analytic semigroup.

Proof of Corollary 2. In view of Theorem 2, it is enough to show that $A(t)$ satisfies $K-T$ condition under the assumption that the bounded operator $A(t) A(s)^{-1}$ is continuously differentiable in the uniform operator topology in $t \in[0, T]$ for any $s$ in $[0, T]$.

Because $\left(A(t) A(s)^{-1}\right)^{-1}=A(s) A(t)^{-1}$, we have

$$
\operatorname{Lim}_{r \rightarrow t} A(s) \frac{A(r)^{-1}-A(t)^{-1}}{r-t}
$$

exists in the uniform operator topology. $A(s)$ being closed, we have that $A(s)\left(d A(t)^{-1} / d t\right)$ is a bounded operator for any $s \in[0, T]$. In particular $A(t)\left(d A(t)^{-1} / d t\right)$ is a bounded operator. Because of the continuous differentiability of $A(s) A(t)^{-1}$, we can find a constant $C$ such that

$$
\begin{array}{cc}
\left\|A(t) \frac{d A(t)^{-1}}{d t}\right\|<C & \text { for } t \in[0, T] . \\
\frac{d}{d t}(A(t)+\lambda)^{-1} & =A(t)(A(t)+\lambda)^{-1} \frac{d}{d t} A(t)^{-1} A(t)(A(t)+\lambda)^{-1} \\
& =(A(t)+\lambda)^{-1} A(t) \frac{d}{d t} A(t)^{-1} A(t)(A(t)+\lambda)^{-1}
\end{array}
$$

therefore

$$
\left\|\frac{d}{d t}(A(t)+\lambda)^{-1}\right\|<\frac{M}{|\lambda|} C(1+M) .
$$

Thus $K-T$ condition is verified with $\rho=0$.

The author acknowledges the encouragement received from Profs. T. Kato and F. Wolf. 


\section{BIBLIOGRAPHY}

1. C. S. Fisher, Accretive operators and an evolution equation in a Banach space, Thesis, University of California, Berkeley, 1963.

2. E. Hille and R. S. Phillips, Functional analysis and semigroups, A.M.S. Coll. Publ. 31 (1957).

3. T. Kato, Integration of the equation of evolution in a Banach space, J. Math. Soc. Japan 5 (1953), 208-234.

4. - On linear differential equations in Banach spaces, Comm. Pure. Appl. Math. 9 (1956), 479-486.

5. - Abstract evolution equations of parabolic type in Banach and Hilbert spaces, Nogoya Math. Journal 19 (1961), 93-125.

6. —_ Perturbation theory, Springer-Verlag, New York, Inc. 1966.

7. - Semigroups and temporally inhomogeneous evolution equations, Lecture notes, Instituto Matematico dell' Universita, Rome.

8. T. Kato and H. Tanabe, On the abstract evolution equation, Osaka. Math. Journal 14 (1962), 107-133.

9. P. E. Sobolevskii, Equations of parabolic type in a Banach space, Trudy Moskov. Mat. Obsc. 10 (1961), 297-350. Math. Rev. 25 (1963), No. 5297.

10. H. Tanabe, $A$ class of the equations of evolution in a Banach space, Osaka Math. J. 11 (1959), 121-145.

11. Remarks on the equations of evolution in a Banach space, Osaka. Math. J. 12 (1960), 145-166.

12. - On the equations of evolution in a Banach space, Osaka Math J. 12 (1960), 363-376.

13. K. Yosida, On the differentiability of semigroups of linear operators, Proc. Japan Acad. 34 (1958), 337-340.

Received March 11, 1965. This is a portion of the author's Ph. D. thesis at the University of California, Berkeley under the direction of Prof. T. Kato. The work on the thesis was supported by N.S.F. Grant No. NSF-G-22982.

The UnIVERSITY OF Kansas

LAWRENCE, KANSAS 



\section{PACIFIC JOURNAL OF MATHEMATICS}

\section{EDITORS}

\section{H. SAMELSON}

Stanford University

Stanford, California

J. P. JANS

University of Washington

Seattle, Washington 98105
J. DUGUNDJI

University of Southern California Los Angeles, California 90007

RICHARD ARENS

University of California

Los Angeles, California 90024

\section{ASSOCIATE EDITORS}
E. F. BECKENBACH
B. H. NEumanN
F. WOLF
K. YosidA

\section{SUPPORTING INSTITUTIONS}

\author{
UNIVERSITY OF BRITISH COLUMBIA \\ CALIFORNIA INSTITUTE OF TECHNOLOGY \\ UNIVERSITY OF CALIFORNIA \\ MONTANA STATE UNIVERSITY \\ UNIVERSITY OF NEVADA \\ NEW MEXICO STATE UNIVERSITY \\ OREGON STATE UNIVERSITY \\ UNIVERSITY OF OREGON \\ OSAKA UNIVERSITY \\ UNIVERSITY OF SOUTHERN CALIFORNIA
}

\author{
STANFORD UNIVERSITY \\ UNIVERSITY OF TOKYO \\ UNIVERSITY OF UTAH \\ WASHINGTON STATE UNIVERSITY \\ UNIVERSITY OF WASHINGTON \\ AMERICAN MATHEMATICAL SOCIETY \\ CHEVRON RESEARCH CORPORATION \\ TRW SYSTEMS \\ NAVAL ORDNANCE TEST STATION
}

Mathematical papers intended for publication in the Pacific Journal of Mathematics should be typewritten (double spaced). The first paragraph or two must be capable of being used separately as a synopsis of the entire paper. It should not contain references to the bibliography. Manuscripts may be sent to any one of the four editors. All other communications to the editors should be addressed to the managing editor, Richard Arens at the University of California, Los Angeles, California 90024.

50 reprints per author of each article are furnished free of charge; additional copies may be obtained at cost in multiples of 50 .

The Pacific Journal of Mathematics is published monthly. Effective with Volume 16 the price per volume ( 3 numbers) is $\$ 8.00$; single issues, $\$ 3.00$. Special price for current issues to individual faculty members of supporting institutions and to individual members of the American Mathematical Society: $\$ 4.00$ per volume; single issues $\$ 1.50$. Back numbers are available.

Subscriptions, orders for back numbers, and changes of address should be sent to Pacific Journal of Mathematics, 103 Highland Boulevard, Berkeley 8, California.

Printed at Kokusai Bunken Insatsusha (International Academic Printing Co., Ltd.), 7-17, Fujimi 2-chome, Chiyoda-ku, Tokyo, Japan.

PUBLISHED BY PACIFIC JOURNAL OF MATHEMATICS, A NON-PROFIT CORPORATION

The Supporting Institutions listed above contribute to the cost of publication of this Journal, but they are not owners or publishers and have no responsibility for its content or policies. 


\section{Pacific Journal of Mathematics

Vol. 22, No. $3 \quad$ March, 1967

Wai-Mee Ching and James Sai-Wing Wong, Multipliers and $H^{*}$

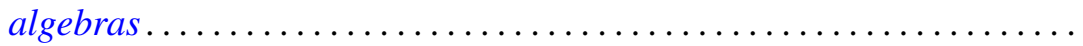

P. H. Doyle, III and John Gilbert Hocking, A generalization of the Wilder

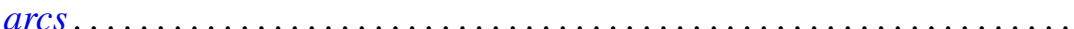

Irving Leonard Glicksberg, A Phragmén-Lindelöf theorem for function

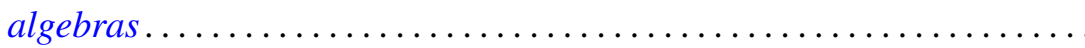

E. M. Horadam, A sum of a certain divisor function for arithmetical semi-groups ..................................... 407

V. Istrăţescu, On some hyponormal operators ................... 413

Harold H. Johnson, The non-invariance of hyperbolicity in partial

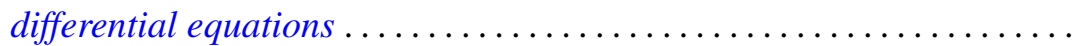

Daniel Paul Maki, On constructing distribution functions: A bounded denumerable spectrum with $n$ limit points................... 431

Ronald John Nunke, On the structure of Tor. II .................... 453

T. V. Panchapagesan, Unitary operators in Banach spaces ............. 465

Gerald H. Ryder, Boundary value problems for a class of nonlinear differential equations ................................. 477

Stephen Simons, The iterated limit condition and sequential convergence .................................... 505

Larry Eugene Snyder, Stolz angle convergence in metric spaces ......... 515 Sherman K. Stein, Factoring by subsets ................... 523

Ponnaluri Suryanarayana, The higher order differentiability of solutions of abstract evolution equations . . .

Leroy J. Warren and Henry Gilbert Bray, On the square-freeness of Fermat and Mersenne numbers ............................... 563

Tudor Zamfirescu, On l-simplicial convexity in vector spaces........... 565 Eduardo H. Zarantonello, The closure of the numerical range contains the spectrum 